Q10 $10 \begin{array}{r}\mathrm{http}: / / \mathrm{d} x . \text { doi.org/10.21611/qirt.2010.144 } \\ 10^{\text {th }} \text { International Conference on Quantitative InfraRed Thermography }\end{array}$

\title{
Narrow-band infrared examination of solid-state structures
}

\author{
by Boris G. Vainer and Gennady N. Kamaev
}

\author{
Rzhanov Institute of Semiconductor Physics, Russian Academy of Sciences, Siberian Branch, Novosibirsk, Russia \\ e-mail: BGV@isp.nsc.ru
}

\begin{abstract}
Successful practical application of narrow spectral range infrared thermography to contactless nondestructive testing of wafer bonding semiconductor structures and power microelectronics devices is demonstrated. It is brought out clearly that bending and nonflatness of silicon wafers as well as bubble-like defects concealed within the wafer interface become accessible to visualisation with the use of a short-wave narrow-band $(2.5-3 \mu \mathrm{m})$ infrared system irrespective to spectral band width of reference light source. Bubble-like defects revealed from thermo-interferograms were verified by means of high-precision grinding of one of the bonded wafers until the real bubbles at the interface become visible. Current-voltage characteristics of unpackaged planar diodes were measured, and peculiarities of current pinching effect were revealed using infrared thermography method. It is shown that infrared thermography is more informative method for testing and presorting of power microelectronics devices than standard electrophysical control.
\end{abstract}

\section{Introduction}

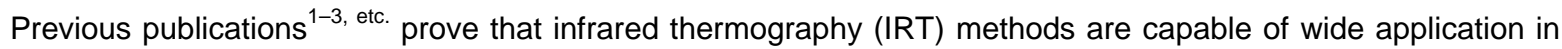
the semiconductor electronics field. In particular, IRT can be highly informative when it comes to defects recognition and determination of local physical characteristics of semiconductor structures and semiconductor-based devices. Our investigations ${ }^{4-6}$ demonstrated that the short-wave narrow-band $(2.5-3 \mu \mathrm{m})$ infrared systems ${ }^{5}$ (SWNBIRSs) are well suited for such measurements. In the present paper, new experimental data obtained with the use of SWNBIRSs are exhibited and discussed.

High-sensitive $\left(0.03{ }^{\circ} \mathrm{C}\right.$ at $\left.30{ }^{\circ} \mathrm{C}\right)$ FPA-based $128 \times 128$ infrared photodetector embedded in SWNBIRS and utilized in our experiments was manufactured with the use of narrow-gap semiconductor indium arsenide ${ }^{7}$. One of the main advantages of SWNBIRS is its narrow working spectral range resulted from the "embedded" in semiconductor structure infrared "natural" narrow-band filter composed of InAs heavily doped substrate and epitaxial photosensitive layer $^{4,5}$. Narrow-spectral-range property of the detector offers the prospect of interferometric analysis to be made with the use of a wide-spectral-range light source ${ }^{4}$ (figure 1 ). Two different optical schemes allowing concealed bubble-like defects located within a double-wafer interface to be recognized and analized are represented in figure 2 . The most important feature displayed in figure 2 is the fact that multiwave interference resulted from the use of nonmonochromatic light source becomes visible due to a separation of a narrow-band spectral interval from mixture of light source electromagnetic waves. This feature can be practically realized owing to the use of a narrow spectral range IR camera. SWNBIRS is in full accord with standards accepted for IRT. It is verified by a great number of our research works $s^{8,9, \text { etc. }}$.

It is well known that wafer direct bonding [10] is a promising technology for the most up-to-date microelectronics and power microelectronics [11]. In particular, this method can be applied sussessfully for fabrication of compound semiconductor structures on silicon wafers $[12,13]$. Industrial use of wafer direct bonding method requires elaboration of technological processes which are capable of providing minimum defects within interface of the bonded wafers. Electrical characteristics and quality of power microelectronics devices depend to a large extent on defects arisen at the bonding interfaces. This is the reason why nondestructive control of the bonding interface and geometrical parameters of primary wafer surfaces (bending, site flatness, etc.) is the problem of vital importance for semiconductor technology.

\section{Sample preparation}

Standard process of bonding structure preparation consists of four major stages: a) wafer chemical treatment to obtain clean and hydrophilic surface; b) prebonding at room temperature in control ambients to join and accommodate two surfaces; c) multistep annealing to obtain covalent siloxane bonds between two wafers; d) thinning of working layer in semiconductor sandwich by grinding and conventional chemical-mechanical polishing to its final thickness. When surfaces are brought into contact, hydrogen bonds begin developing. When heated up to $200{ }^{\circ} \mathrm{C}$, the molecules of adsorbed water form a common water cluster consisting of four molecules. At $700{ }^{\circ} \mathrm{C}$ the cluster decays and water molecules diffuse away from the interface. At $1100^{\circ} \mathrm{C}$ additional siloxane bonds begin forming due to water leaving. Due to diffusion of atoms along the interface and viscosity of native oxide, microcavities become filled [14].

Experimental structures were formed by direct bonding of $(100)$ silicon wafers at $1100{ }^{\circ} \mathrm{C}$ for 2 hours in nitrogen ambient. Prior to bonding, the wafers were given a standard RCA cleaning procedure, water rinse, and drying on a spin drier. Two wafers were put into face-to-face intimate contact in cleanroom ambient at room temperature. Then external pressure was applied homogeneously across the entire wafer area. 


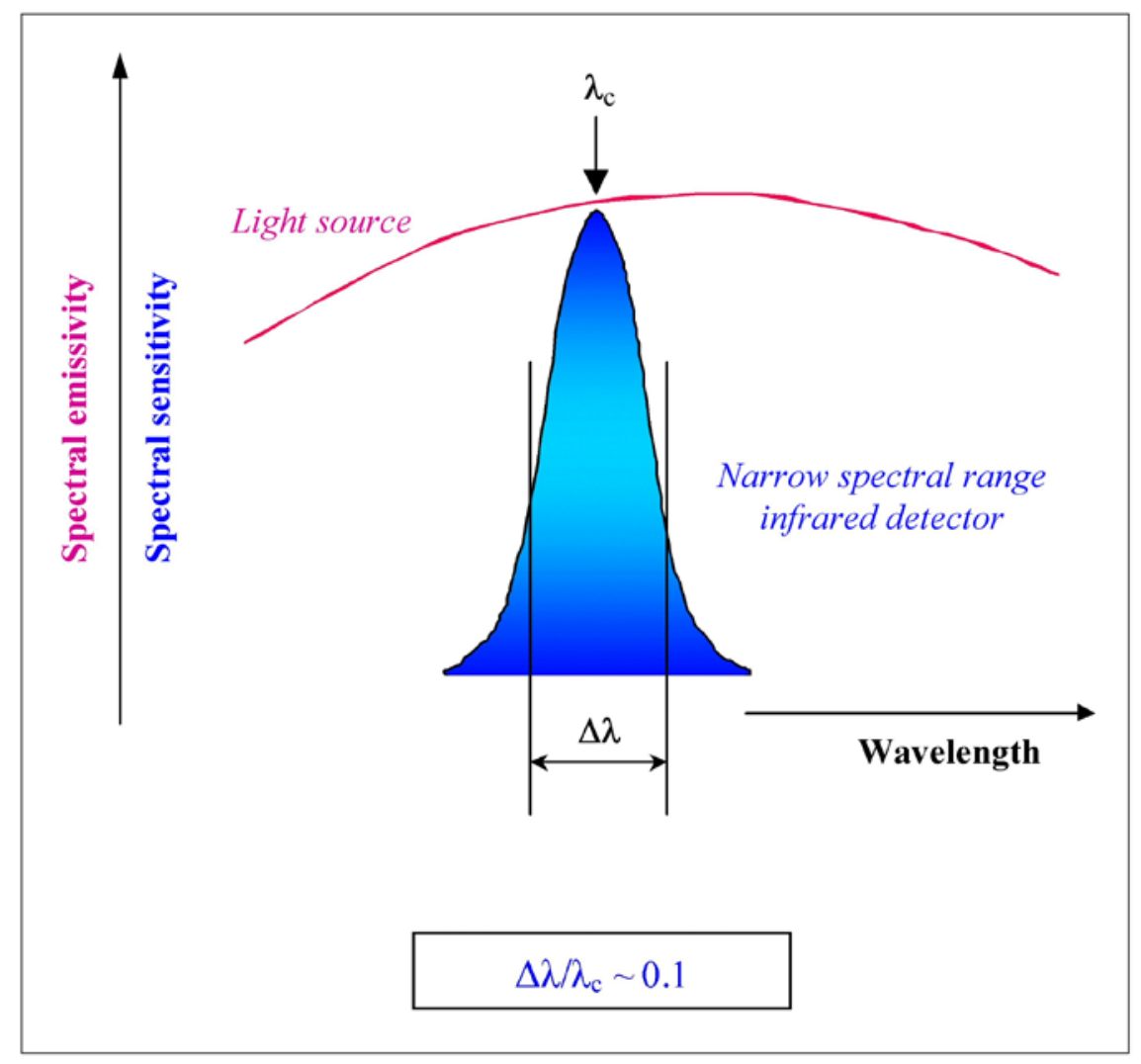

Fig. 1. Schematic representation of simultaneous use of arbitrary light source and narrow spectral range infrared detector. Shown are: $\lambda_{c}$ - characteristic operating wavelength of the narrow-band detector, $\Delta \lambda$ - working spectral range of the detector.

Diodes were fabricated using both the standard diffusion doping (round samples) and wafer bonding (hexahedral and square samples) technologies.

\section{Experimental results}

Figure 3 demonstrates how the bending of silicon wafer is represented in the thermo-interferogram obtained with SWNBIRS. Interference pattern appears as a result of a gap between flat metallized glass plate and silicon wafer. Thermal radiation emitted from human hand served as the reference light.

Another example of interferometric measurements is given in figure 4. A set of thermo-interferograms shows how the small local voids located between two bonded silicon wafers evolve in the course of structure annealing. In this instance, SWNBIRS-assisted IRT furnishes insights into the nature of concealed defects hidden in the silicon-silicon interface. Figure 5 confirms that interference pattern represented in thermo-interferograms is really determined by bubbles and gaps existing in the bonding interface region. Photograph of the ground structure demonstrates clearly the bubbles located at the same places as interferometric rounds.

With the use of SWNBIRS, we brought to light hidden physical processes (breakdown and leakage current) in the above-mentioned unpackaged high-voltage diodes of different dimension types. At high voltages, current crowding at the area of reverse-biased diodes was observed (figure 6). Bright spots at $600 \mathrm{~V}$ and $1800 \mathrm{~V}$ appeared due to microplasma and indicated of the diode breakdown. Two thermograms in the bottom of figure 3 demonstrate an abrupt generation of second pinch within the diode area.

It was found that a leakage current accompanies the local temperature increase (figure 7). It should be noted that the described effect can not be satisfactorily explained with invoking the radiative recombination mechanism due to insensibility SWNBIRS to wavelengths emitted by silicon. Figure 7 demonstrates that semiconductor technology might benefit from the use of IRT-defectoscopy method. In addition to standard information coming from current-voltage characteristic measurement, IRT allows mapping the defects. The latter is highly important because the analysis of current pinches localities carries valuable information to technologists. 


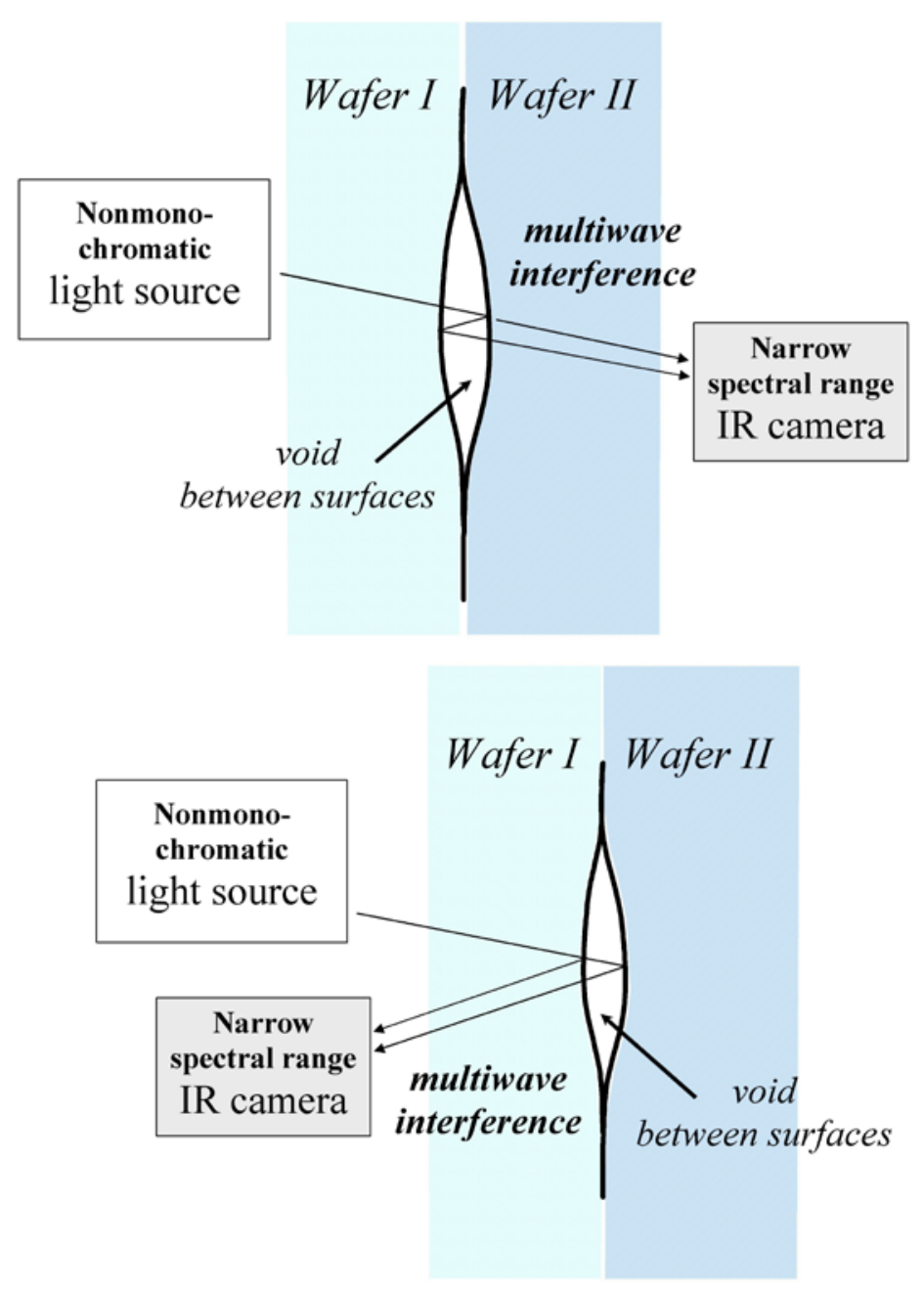

Fig. 2. Transmittance- (on top) and reflectance- (in the bottom) optical schemes used for defects recognition in multilayer semiconductor structures (two bonded semiconductor wafers are shown).

\section{REFERENCES}

[1] Wiecek B., Grecki M., Pacholik J. Thermal measurements of power semiconductor devices using thermographic system In: Quantitative InfraRed Thermography QIRT 92, Eurotherm Seminar 27, Chatenay-Malabry, France, July 7-9, 1992. Proceedings. Ed. by D.Balageas, G.Busse, and G.M.Carlomagno. ONERA, p. 291-295, 1992..

[2] Wiecek B., Michalski A., Napiorkowski K. IR microscope measurement of the plasma spreading in thyristors. In: Quantitative InfraRed Thermography 4, Eurotherm Seminar 60, QIRT’98, Lodz, Poland, September 7-10, 1998. Proceedings. Ed. by D.Balageas, G.Busse, and G.M.Carlomagno. PKOpto SEP, p. 191-194, 1998.

[3] Rossi L., Breglio G., Irace A., Spirito P. Thermal transient mapping systems for integrated semiconductor devices and circuits, In: $8^{\text {th }}$ conference on Quantitative InfraRed Thermography, QIRT 2006, Padova, Italy, June 27-30, 2006. Book of Abstracts. CNR ITC, p. 23-24, 2006.

[4] Vainer B.G., Kamaev G.N., Kurishev G.L. Application of the narrow spectral range InAs-FPA-based IR camera for the investigation of the interface voids in silicon wafer bonding. J. Cryst. Growth, 210, p. 351-355, 2000.

[5] Vainer B.G. Narrow spectral range infrared thermography in the vicinity of $3 \mu \mathrm{m}$ operating wavelength. In: Quantitative InfraRed Thermography 5, Eurotherm Seminar 64, QIRT’2000, Reims, France, July 18-21, 2000. Proceedings. Ed. by D.Balageas, J.-L.Beaudoin, G.Busse, and G.M.Carlomagno. UTAP URCA, p. 84-91, 2000.

[6] Kamaev G.N., Vainer B.G. Infrared imaging control at different steps of semiconductor devices fabrication. - In: 6-th International Workshop on Expert Evaluation \& Control of Compound Semiconductor Materials \& Technologies, EXMATEC 2002, Budapest, Hungary, 26-29 May 2002. Book of Abstracts. Hungary, p. 69, 2002. 
[7] Kuryshev G.L., Kovchavtzev A.P., Vainer B.G., Guzev A.A., Bazovkin V.M., Stroganov A.S., Subbotin I.M., Zakharov I.M., Efimov V.M., Postnikov K.O., Lee I.I., Valisheva N.A., Panova Z.V. Medical infrared imaging system based on a $128 \times 128$ focal plane array for 2.8-3.05 $\mu \mathrm{m}$ spectral range, Optoel., Instrum. and Data Proces. (Autometria), 4, p. 5-10, 1998.

[8] Vainer B.G. FPA-based infrared thermography as applied to the study of cutaneous perspiration and stimulated vascular response in humans, Phys. Med. Biol., 50, p. R63-R94, 2005.

[9] Vainer B.G. Focal plane array based infrared thermography in fine physical experiment, J. Phys. D: Appl. Phys., 41, p. 065102 (12 pp), 2008.

[10] Shimbo M., Furukawa K., Fukuda K., Tanzawa K. Silicon-to-Silicon direct bonding method, J. Appl. Phys., 60, p. 2987-2989, 1986.

[11] Tong Q.-Y., Gosele U. Semiconductor Wafer Bonding: Science and Technology. N.-Y. Wiley-Interscience Publication, 1999. $297 \mathrm{pp}$.

[12] Lehmann V., Mitani K., Stengl R., Mii T., Gosele U. Bubble-free wafer bonding of GaAs and InP on silicon in a microcleanroom, Japan. J. Appl. Phys., 28, L2141-2143, 1989.

[13] Kastner G., Akatsu T., Senz S., Plosl A., Gosele U. Large-area wafer bonding of GaAs using hydrogenand ultrahigh vacuum atmospheres, Appl. Phys. A: Mat. Sci. Proc., 70, p.13-19, 2000.

[14] Stengl R., Tan T., Gosele U. A Model for the silicon wafer bonding process, Japan. J. Appl. Phys., 28, p. 1735-1741, 1989.

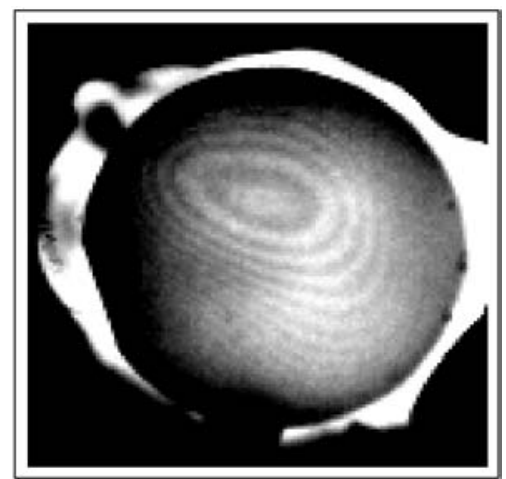

Fig. 3. Silicon wafer bending represented by thermo-interferogram measured with SWNBIRS. Silicon wafer was placed on extremely flat metallized glass. IR camera detects the beams emitted by human hand (infrared radiation source) and reflected from metal.

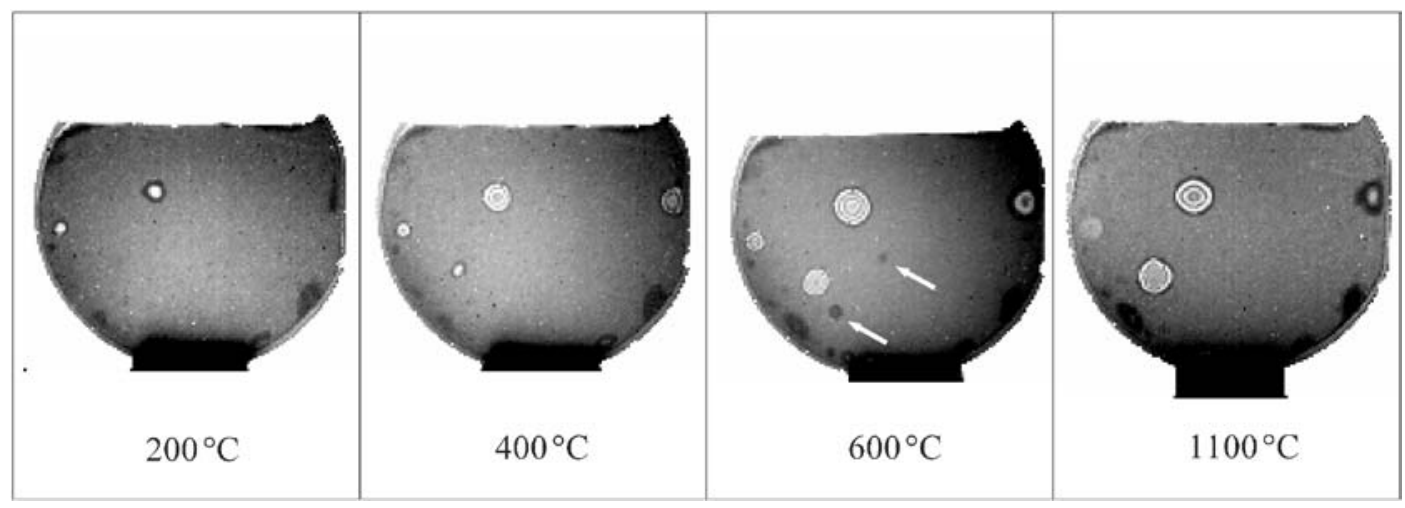

Fig. 4. Evolution of interface defects (thin bubbles) concealed between two bonded silicon wafers. Legends under thermograms display the annealing temperature $T_{a}$. Arrows indicate the nuclei of two virtual defects arisen at $T_{a}=600{ }^{\circ} \mathrm{C}$ and disappeared at higher temperature. 


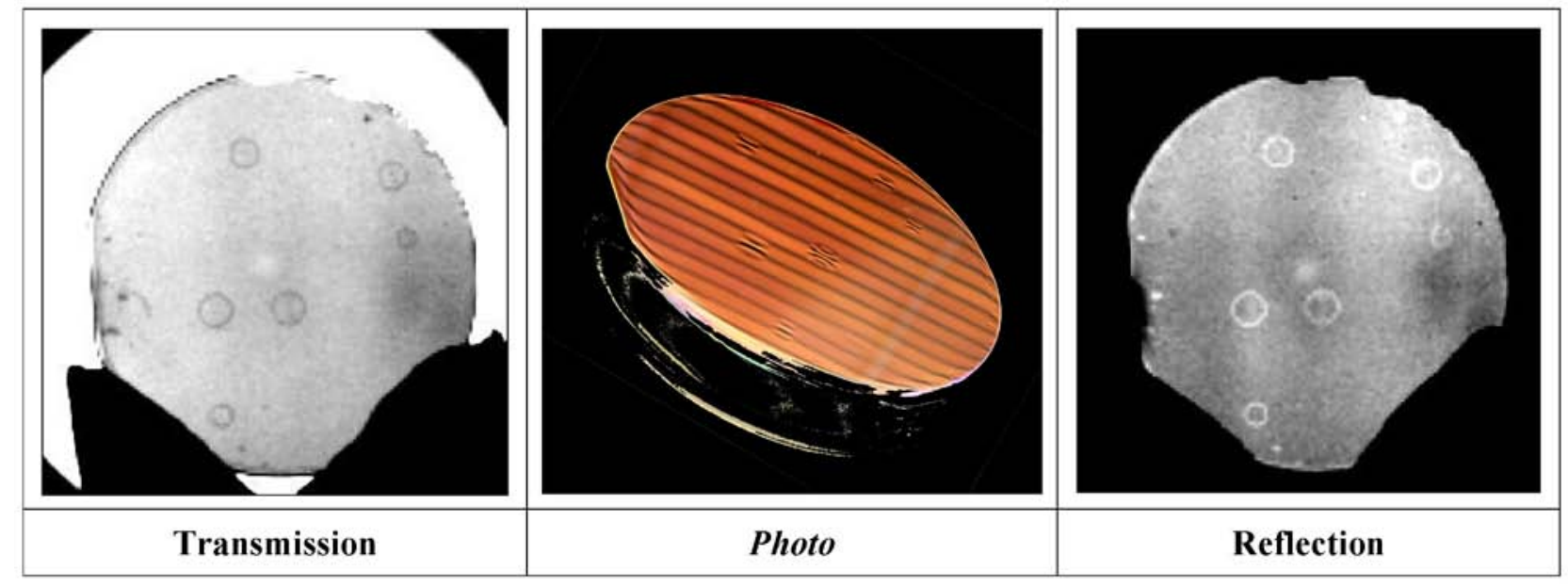

Fig. 5. Thermograms (on the left and on the right) and the corresponding photograph (in the middle) of a silicon structure consisted of two bonded wafers; one of the wafers (upper) was mechanically grinded and polished until the interface bubbles become visible. Stripes in the photo are due to window jalousie reflected from silicon surface (with this reflection, the bulging bubbles are seen distinctly). Left thermogram is obtained with transmittance optical scheme, right - with reflectance scheme.

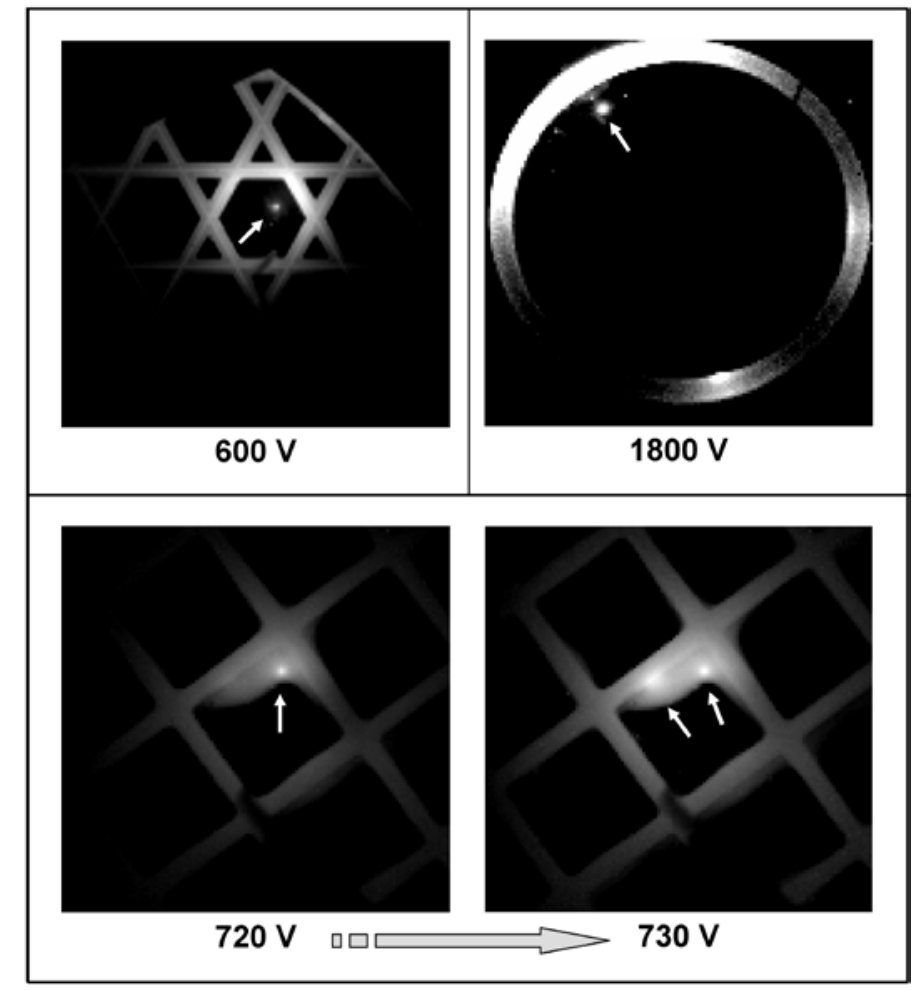

Fig. 6. Thermograms of the reverse-biased high-voltage diodes with current leakage (arrows). Legends are the external voltages applied across the $p-n$ junctions. 


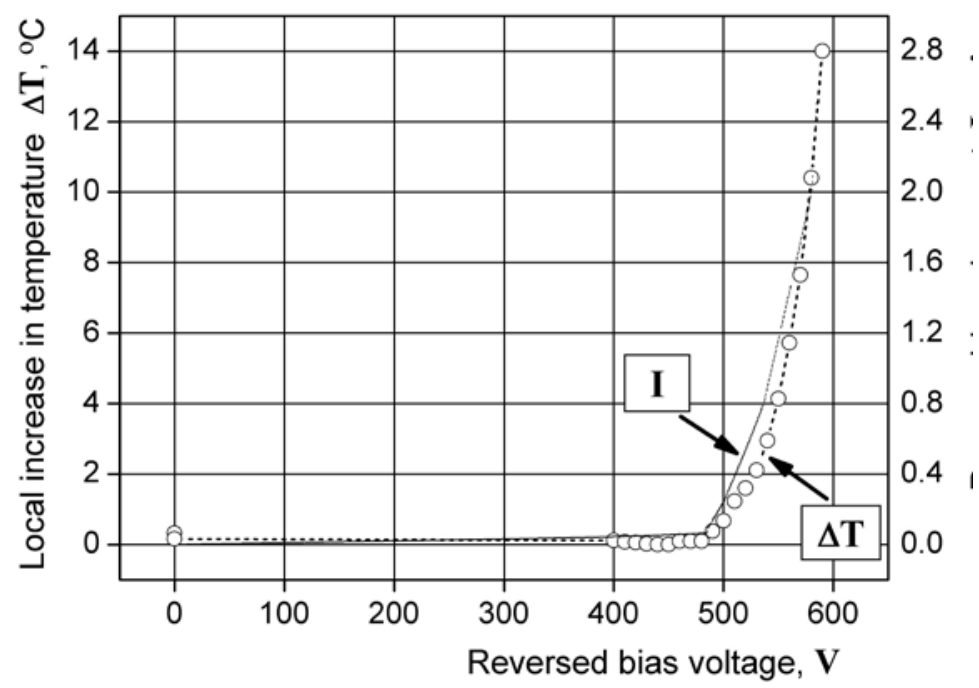

Fig. 7. Comparison of the diode current-voltage and temperaturevoltage characteristics. 